\title{
The importance of central airway dilatation in patients with bronchiolitis obliterans
}

\author{
Mariko Kogo ${ }^{1}$, Hisako Matsumoto ${ }^{1,5}$, Naoya Tanabe $\mathbb{1}^{1,5}$, Toyofumi F. Chen-Yoshikawa ${ }^{2}$, Naoki Nakajima ${ }^{3}$, \\ Akihiko Yoshizawa ${ }^{3}$, Tsuyoshi Oguma ${ }^{1}$, Susumu Sato $\mathbb{D}^{1}$, Natsuko Nomura ${ }^{1}$, Chie Morimoto ${ }^{1}$, \\ Hironobu Sunadome ${ }^{1}$, Shimpei Gotoh ${ }^{1}$, Akihiro Ohsumi ${ }^{4}$, Hiroshi Date $\mathbb{C}^{4}$ and Toyohiro Hirai ${ }^{1}$
}

${ }^{1}$ Dept of Respiratory Medicine, Graduate School of Medicine, Kyoto University, Kyoto, Japan. ${ }^{2}$ Dept of Thoracic Surgery, Graduate School of Medicine, Nagoya University, Nagoya, Japan. ${ }^{3}$ Dept of Diagnostic Pathology, Graduate School of Medicine, Kyoto University, Kyoto, Japan. ${ }^{4}$ Dept of Thoracic Surgery, Graduate School of Medicine, Kyoto University, Kyoto, Japan. ${ }^{5}$ These authors contributed equally to this work.

Corresponding author: Hisako Matsumoto (matsumoto.hisako.v92@kyoto-u.jp)

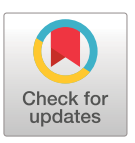

Copyright $\odot$ The authors 2021

This version is distributed under the terms of the Creative Commons Attribution Non-Commercial Licence 4.0. For commercial reproduction rights and permissions contact permissions@ersnet.org

This article has supplementary material available from openres.ersjournals.com

Received: 7 Feb 2021

Accepted: 2 June 2021

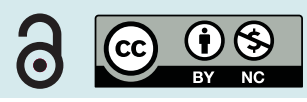

Shareable abstract (@ERSpublications)

In patients with bronchiolitis obliterans (BO), airway dilatation may reflect air trapping and the pathological extent of obstructive bronchioles. More attention should be paid to airway dilatation in the management of BO. https://bit.ly/3w7cRV6

Cite this article as: Kogo $\mathrm{M}$, Matsumoto $\mathrm{H}$, Tanabe $\mathrm{N}$, et al. The importance of central airway dilatation in patients with bronchiolitis obliterans. ERJ Open Res 2021; 7: 00123-2021 [DOI: 10.1183/ 23120541.00123-2021].

\section{Abstract}

Background Bronchiolitis obliterans (BO) is a clinical syndrome characterised by progressive small airway obstruction, causing significant morbidity and mortality. Central airway dilatation is one of its radiological characteristics, but little is known about the clinical and pathological associations between airway dilatation and $\mathrm{BO}$.

Methods This retrospective study consecutively included patients who underwent lung transplantation due to BO at Kyoto University Hospital from 2009 to 2019. Demographic and histopathological findings of the resected lungs were compared between patients with and without airway dilatation measured by chest computed tomography (CT) at registration for lung transplantation.

Results Of a total of 38 included patients (median age, 30 years), 34 (89\%) had a history of hematopoietic stem-cell transplantation, and 22 (58\%) had airway dilatation based on CT. Patients with airway dilatation had a higher frequency of Pseudomonas aeruginosa isolation with greater residual volume than those without airway dilatation. Quantitative CT analysis revealed an increase in lung volume to predictive total lung capacity and a percentage of low attenuation volume $<-950 \mathrm{HU}$ at inspiration in association with the extent of airway dilatation. Airway dilatation on CT was associated with an increased number of bronchioles with concentric narrowing of the lumen and thickening of the subepithelium of the walls on histology.

Conclusions In patients with BO, airway dilatation may reflect increased residual volume or air trapping and pathological extent of obstructive bronchioles, accompanied by a risk of Pseudomonas aeruginosa isolation. More attention should be paid to the development of airway dilatation in the management of $\mathrm{BO}$.

\section{Introduction}

Bronchiolitis obliterans (BO) is a form of irreversible airflow obstruction following an injury to the respiratory and terminal bronchioles from various potential causes [1-3]. BO is rare, but is becoming more frequent as a complication of hematopoietic stem-cell transplantation (HSCT) and lung transplantation [2]. Although the impact of BO on post-transplantation prognosis is milder than that of restrictive forms, BO typically progresses and eventually causes death or requires lung transplantation [1-4].

Currently, the presence and progression of BO are primarily defined by spirometry. However, many studies have emphasized the usefulness of radiological findings [5-9]. In addition to the low attenuation area at a single expiratory image, the recent development of computed tomography (CT) analysis has enabled the 
quantitative evaluation of air trapping separately from emphysema by comparing the inspiratory and expiratory images on a voxel-by-voxel basis with a nonrigid registration technique or parametric response mapping $[6,7,10]$.

Central airway dilatation, another radiological characteristic of BO, is less likely to receive attention than air trapping. In the 1990s, several studies focused on central airway dilatation and its association with airflow limitation in BO [11-14]. Although the sensitivity and specificity of central airway dilatation to detect the early phase of $\mathrm{BO}$ were lower than those of air trapping, several studies have mentioned that central airway dilatation is one of the critical manifestations for detecting the development of BO [5, 15]. Furthermore, because the central airway dilatation is easily detectable on inspiratory CT, it might be informative during the follow-up of BO patients, i.e. not only for detecting BO. Despite its potential roles in the management of $\mathrm{BO}$, studies on the clinical impacts of central airway dilatation and its association with pathological findings of BO are limited [16]. This study revealed the underlying clinical, radiological and pathological aspects related to airway dilatation among BO patients.

Materials and methods

Study design and subjects

We conducted a historical cohort study enrolling patients who underwent lung transplantation due to BO that was pathologically confirmed with samples taken during lung transplantation at the Kyoto University Hospital between January 2009 and April 2019 (figure 1). The pathological definition of BO is the existence of one or more bronchioles with concentric narrowing of the bronchiolar lumen by inflammation and fibrosis [1]. Demographic, clinical, respiratory functional and radiological data at registration for lung transplantation were retrieved from clinical records. Bacterial cultures were evaluated with the spontaneous sputum at the time of registration when patients did not exhibit signs of acute infection. Standard laboratory protocols were used to identify the pathogenic bacterial species that colonise the lower respiratory tract. Microbiological analysis was not performed on lung tissue obtained at the time of transplantation. When available, clinical and respiratory functional data and CT findings between registration and the transplantation were also collected. Patients were excluded from the quantitative CT analysis when they lacked high-resolution computed tomography (HRCT) in defined reconstruction (Supplementary methods), were on ventilator support, or had a pneumothorax (figure 1). Written informed consent for the examination was obtained from each patient at the time of lung transplantation for research using their clinical data and histological samples. The Ethics Committee of Kyoto University approved the study (approval no. G0469).

\section{CT analysis}

Definition of central airway dilatation

Details of CT and histopathological analyses are described in the Supplementary methods.

HRCT scanning was acquired at the time of registration for lung transplantation. On full-inspiratory CT, airway dilatation was determined when at least one of the following criteria was fulfilled, independently of interstitial lung abnormalities [17]: 1) internal diameter of the bronchus greater than that of the adjacent pulmonary artery; and 2) lack of tapering of the bronchial lumen toward the periphery for any generation. The extent of airway dilatation was semi-quantitatively assessed using a modified Reiff score (Supplementary methods) [18].

\section{Quantitative CT analysis}

The total lung volume (CT-TLV) and the percentage of low attenuation volume $<-950$ Hounsfield units (HU) (LAV_-950\%) were evaluated on full-inspiratory CT. The predicted total lung capacity (pred TLC) was calculated from the sex and height, and used to adjust the CT-TLV by the natural size of the lungs [19]. In a subgroup of patients whose full-expiratory CT scans were available, the percentage of low attenuation volume $<-856 \mathrm{HU}$ at expiration $\left(\mathrm{LAV}_{-856} \%\right.$ ) was calculated to evaluate air trapping. Additionally, nonemphysematous air trapping, presumably a surrogate of small airway disease severity, termed functional small airway disease (fSAD) [20], was calculated by nonrigidly registering inspiratory and expiratory CT scans using custom software.

For the evaluation of longitudinal CT data before and after the clinical diagnosis of BO in representative patients, the lumen of a branch from the basal posterior bronchus $\left(\mathrm{B}^{10} \mathrm{a}\right)$ was measured as reported previously [15]. 


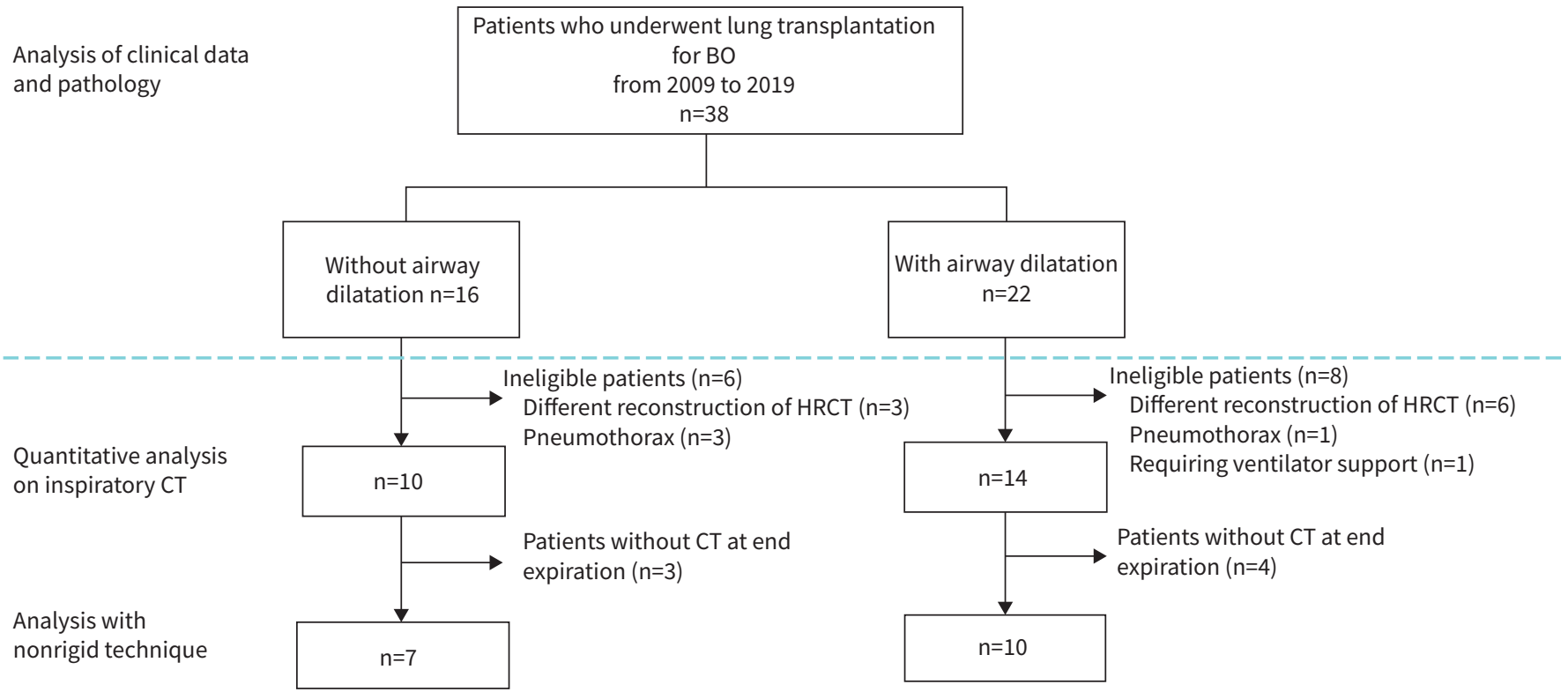

FIGURE 1 Patient flow chart. Thirty-eight patients were included, of which 22 had airway dilatation on CT. Twenty-four and 17 patients were quantitatively analysed for inspiratory $\mathrm{CT}$ and paired inspiratory-expiratory $\mathrm{CT}$, respectively. BO: bronchiolitis obliterans; CT: computed tomography; HRCT: high-resolution computed tomography.

\section{Histopathological analysis}

The lung tissue obtained at the time of lung transplantation was evaluated. Lungs were inflated with undetermined pressure and fixed by formalin, processed into paraffin blocks, and cut into a section of 4- $\mu \mathrm{m}$ thickness according to routine surgical pathology protocols [21]. According to a previous study [22], the six sections from affected lungs containing one or more bronchioles were stained with haematoxylin and eosin and either elastica van Gieson or elastica Masson. On digital images of whole sections [23], relatively circular bronchioles (long axis/short axis <3) smaller than $2 \mathrm{~mm}$ in long axis were evaluated [22, 24], and the perimeter of the basement membrane defined as an elastic layer of the bronchiole (Pbm) was measured. The airway wall thickness of three compartments, including the epithelium, subepithelium (from the bottom of the epithelium to the basement membrane) and lamina propria (from the basement membrane to the outer edge of the smooth muscle), was assessed by dividing each area by Pbm (Suppl. Figure 1) [22]. Bronchioles with concentric narrowing of the bronchiolar lumen by inflammation and fibrosis were identified. The percentage of the number of these bronchioles to the total number of bronchioles evaluated on sections was measured for each patient to evaluate the pathological extent of BO. The number of alveolar attachments to the outer wall of bronchioles was counted and adjusted by Pbm.

\section{Statistical analysis}

The patients with BO were divided into those with and without airway dilatation determined by CT and compared. Continuous variables were compared using the Wilcoxon rank-sum test, and categorical variables were compared using the Pearson chi-square test. The correlations between Reiff score and quantitative CT analysis variables or histological analysis were presented as Spearman correlation coefficients. The threshold of Reiff score in relation to the extent of pathological BO (the cut-off value was set at the first tertile in our cohort) was estimated using a receiver operating characteristic (ROC) curve analysis. A two-tailed p-value $<0.05$ was considered statistically significant. All statistical analyses were performed using JMP Pro 14 for Windows (SAS Institute, Inc., Cary, NC). Data are presented as median values with interquartile ranges (IQR) for continuous variables and as percentages for categorical variables.

\section{Results}

\section{Patient characteristics}

During the study period, 38 patients underwent lung transplantation due to BO (figure 1). The main cause of BO was HSCT (89\%), followed by lung transplantation (11\%). The median time from the clinical diagnosis of $\mathrm{BO}$ to lung transplantation registration and from registration to lung transplantation was 2.1 years and 1.6 months, respectively. Twenty-nine patients (76\%) received living-donor lung 
transplantation. The patients' clinical features are summarised in table 1. Among these cases, 22 (58\%) had developed airway dilatation (Reiff score $\geqslant 1$ ) at the time of registration for lung transplantation, with a median Reiff score of 7. Among the 22 cases, HRCT at the diagnosis of BO was available for seven patients and two patients had already developed airway dilatation at the time of the diagnosis. In 21 patients whose pulmonary function was available, patients with airway dilatation $(n=12)$ had a higher residual volume (RV) and a tendency toward higher RV/TLC than those without airway dilatation $(n=9)$. However, $\mathrm{FEV}_{1}$ did not differ between the two groups. The time from diagnosis of $\mathrm{BO}$ to registration was not significantly different between patients with and without airway dilatation among patients who had pulmonary function data $(p=0.48)$ as well as those of the total cohort. Sputum culture was obtained for 32 patients. Patients with airway dilatation $(n=18)$ had a higher frequency of detection of Pseudomonas aeruginosa (table 2).

\section{Quantitative CT analysis}

Quantitative CT analysis was performed in 24 patients (figure 1). On inspiratory CT, patients with airway dilatation $(\mathrm{n}=14)$ had greater CT-TLV/pred TLC and $\mathrm{LAV}_{-950} \%$ than those without airway dilatation $(n=10)$ (table 3A). Moreover, there were significant correlations between Reiff score and CT-TLV/pred TLC, $\mathrm{LAV}_{-950} \%$ (rho=0.64, $\mathrm{p}<0.001$ and $\mathrm{rho}=0.45, \mathrm{p}=0.029$, respectively) (figure $2 \mathrm{a}$ and b). A representative CT image of a BO patient with high $\mathrm{LAV}_{-950} \%$ is shown in Suppl. Figure 2A, B. Analysis using expiratory CT $(n=17)$ revealed significantly higher $\mathrm{LAV}_{-856} \%$ and a trend for increased fSAD in patients with airway dilatation $(n=10)$ than those without airway dilatation $(n=7)$ (table $3 B)$. Finally, the Reiff score was marginally correlated with fSAD (rho=0.48, $\mathrm{p}=0.053$ ).

Longitudinal data from the onset of BO were available for three patients, all of whom developed BO after lung transplantation. Pulmonary function tests were discontinued due to recurrent pneumothorax. A patient with airway dilatation (figure 3a-d) showed rapid disease progression, expressed as an increase in RV/TLC

TABLE 1 Patient characteristics at registration for lung transplantation

\begin{tabular}{|c|c|c|c|}
\hline & $\begin{array}{l}\text { Airway dilatation- } \\
\qquad \mathrm{n}=16\end{array}$ & $\begin{array}{l}\text { Airway dilatation+ } \\
\qquad \mathrm{n}=22\end{array}$ & $p$-value \\
\hline Male & $9(56)$ & $9(41)$ & 0.35 \\
\hline Age, years & $33(14-47)$ & $25(15-42)$ & 0.43 \\
\hline Height, cm & $158(141-169)$ & $159(143-164)$ & 0.89 \\
\hline Weight, kg & $39.3(25.7-50.6)$ & $40.0(28.9-49.6)$ & 0.70 \\
\hline Ex-smokers & $1(6)$ & $3(14)$ & 0.46 \\
\hline \multicolumn{4}{|l|}{ Causes of BO } \\
\hline $\mathrm{HSCT}$ & $14(88)$ & $20(91)$ & 0.74 \\
\hline Lung transplantation & $2(13)$ & $2(9)$ & \\
\hline Bilateral/right/left & $0(0) / 1(6) / 1(6)$ & $2(9) / 0(0) / 0(0)$ & \\
\hline BO with prominent fibrosis & $3(19)$ & $1(5)$ & 0.16 \\
\hline Prednisolone use & $11(69)$ & $19(86)$ & 0.19 \\
\hline Dose, $\mathrm{mg} \cdot \mathrm{day}^{-1}$ & $15(7.5-15)$ & $8(4.0-12.5)$ & 0.13 \\
\hline Immunosuppressive agent use & $6(38)$ & $12(55)$ & 0.30 \\
\hline Inhaled corticosteroid use & $10(63)$ & $12(55)$ & 0.62 \\
\hline Time from the causes of $\mathrm{BO}$ to diagnosis of $\mathrm{BO}$, year & $2.3(0.8-5.2)$ & $1.3(0.7-2.9)$ & 0.17 \\
\hline Time from the diagnosis of $\mathrm{BO}$ to registration, years & $1.5(0.6-4.1)$ & $2.8(1.1-6.0)$ & 0.15 \\
\hline Age at diagnosis of $\mathrm{BO}$, years & $31(13-44)$ & $21(12-37)$ & 0.31 \\
\hline Time from registration to lung transplantation", months & $2.4(0.6-18.8)$ & $1.5(1.2-17.3)$ & 0.73 \\
\hline \multicolumn{4}{|l|}{ Pulmonary function test } \\
\hline FVC, \% of predicted & $48.3(16.3-68.4)$ & $46.5(38.9-50.8)$ & 1.00 \\
\hline $\mathrm{FEV}_{1}, \%$ of predicted & $20.1(16.7-44.0)$ & $22.0(18.3-23.0)$ & 0.76 \\
\hline $\mathrm{FEV}_{1} / \mathrm{FVC}, \%$ & $68.5(35.0-96.1)$ & $42.7(34.5-50.1)$ & 0.15 \\
\hline $\mathrm{RV}, \%$ of predicted & $87.0(54.6-166.2)$ & $176.8(131.7-236.1)$ & 0.03 \\
\hline $\mathrm{RV} / \mathrm{TLC}, \%$ & $46.4(41.9-50.8)$ & $53.2(46.9-58.9)$ & 0.12 \\
\hline Pneumonia within the previous year & $6(38)$ & $8(36)$ & 0.94 \\
\hline Reiff score & 0 & $7(4-10)$ & - \\
\hline \multicolumn{4}{|c|}{$\begin{array}{l}\text { BO: bronchiolitis obliterans; HSCT: hematopoietic stem-cell transplantation; FVC: forced vital capacity; FEV }{ }_{1} \text { : } \\
\text { forced expiratory volume in } 1 \mathrm{~s} \text {; RV: residual volume; TLC: total lung capacity. All values are expressed as } \\
\text { median (interquartile range) except categorical variables, which are expressed as } n(\%) \text {. } 29 \text { patients received } \\
\text { living-donor lung transplantation. "Available for } 21 \text { patients (airway dilatation+ } n=12 \text {, airway dilatation- } n=9 \text { ). }\end{array}$} \\
\hline
\end{tabular}


TABLE 2 Bacterial culture of the sputum at registration

\begin{tabular}{lccc} 
& $\begin{array}{c}\text { Airway dilatation- } \\
\mathrm{n}=\mathbf{1 4}\end{array}$ & $\begin{array}{c}\text { Airway dilatation+ } \\
\mathrm{n}=\mathbf{1 8}\end{array}$ & p-value \\
\hline Streptococcus ssp. & $0(0)$ & $1(6)$ & 0.37 \\
\hline Staphylococcus ssp. & $0(0)$ & $2(11)$ & 0.20 \\
\hline Pseudomonas aeruginosa & $0(0)$ & $7(39)$ & 0.01 \\
\hline Other gram-negative bacilli & $2(14)$ & $3(17)$ & 0.85 \\
\hline Haemophilus influenzae & $1(7)$ & 0 & \\
Klebsiella oxytoca & $1(7)$ & 0 & 0.02 \\
\hline Escherichia coli & 0 & $1(11)$ & 0.37 \\
\hline Stenotrophomonas maltophilia & 0 & $8(44)$ & $1(6)$ \\
\hline Normal flora only & $12(86)$ & & \\
\hline No growth & $0(0)$ & & \\
\hline All values are expressed as $\mathrm{n}(\%)$. & & & \\
\hline
\end{tabular}

and areas of fSAD in the following year after diagnosis of BO. In contrast, the clinical course of the patient without airway dilatation (figure $3 e$ and $\mathrm{f}$ ) was relatively stable during the same period.

\section{Pathological analysis}

The number of bronchioles examined per patient and their mean Pbm were similar between patients with and without airway dilatation recognised on CT (Suppl. Figure 3A, 3B). As shown in figure 4a, obstructive bronchioles on histology were more frequently found in patients with airway dilation than in those without (median [IQR] 65\% [38-80] versus 19\% [5-39], $\mathrm{p}<0.001$ ). This difference was still significant even when adjusted for the time from diagnosis of $\mathrm{BO}$ to lung transplantation registration $(\mathrm{p}=0.002)$. Obstructive bronchioles contained foamy macrophages (Suppl. Figure 1) as well as the deposition of extracellular matrix in the subepithelium (figure 3c and 3d) when compared with normal bronchioles (figure $3 \mathrm{~g}$ and $3 \mathrm{~h}$ ). In patients with airway dilatation, the thicknesses of subepithelium, but not the lamina propria, were significantly greater, while that of the epithelium was smaller than those without airway dilatation (figure 4b). Additionally, there were positive correlations between the Reiff score on CT and the pathological extent of BO or thickness of subepithelium (rho=0.55, $\mathrm{p}<0.001$ and $r h o=0.57, \mathrm{p}<0.001$, respectively) (figure $4 \mathrm{c}$ and $4 \mathrm{~d}$ ). The first tertile of the extent of BO in our cohort was $62 \%$, and ROC analysis of Reiff score for this value showed an area under the curve (AUC) of 0.73 with the best cut-off value of Reiff score of 1 (sensitivity 0.92 , specificity 0.60 ). The number of alveolar attachments per Pbm was comparable between the groups (Suppl. Figure 3C). On the pathological observation on dilated central airways, airway dilatation was not accompanied by traction ectasia due to fibrosis (Suppl. Figure 4). To minimise the effect of the period between data at registration and transplantation when the pathologies were collected, we limited the analysis to patients who received living-donor lung transplantation, which yielded similar results to those of the whole patients (Suppl. Figure 5).

TABLE 3 Quantitative analysis for inspiratory CT (A) and paired inspiratory and expiratory CT (B) among patients with and without airway dilatation

\begin{tabular}{|c|c|c|c|}
\hline & $\begin{array}{l}\text { Airway dilatation- } \\
\qquad \mathrm{n}=10\end{array}$ & $\begin{array}{l}\text { Airway dilatation+ } \\
\qquad \mathrm{n}=14\end{array}$ & $\mathrm{p}$-value \\
\hline CT-TLV/pred TLC, \% & $42.0(21.3-58.9)$ & $69.7(56.7-97.0)$ & 0.009 \\
\hline LAV $_{-950} \%, \%$ & $15.3(5.7-25.1)$ & $29.3(15.1-41.7)$ & 0.050 \\
\hline \multicolumn{4}{|l|}{ B } \\
\hline & $\begin{array}{l}\text { Airway dilatation- } \\
\qquad \mathrm{n}=7\end{array}$ & $\begin{array}{l}\text { Airway dilatation+ } \\
\qquad \mathrm{n}=10\end{array}$ & $\mathrm{p}$-value \\
\hline $\mathrm{LAV}_{-856} \%, \%$ & $46.3(0.6-57.2)$ & $66.5(54.1-72.6)$ & 0.036 \\
\hline fSAD, $\%$ & $10.0(0.2-33.9)$ & $34.7(28.3-36.4)$ & 0.057 \\
\hline
\end{tabular}


a)

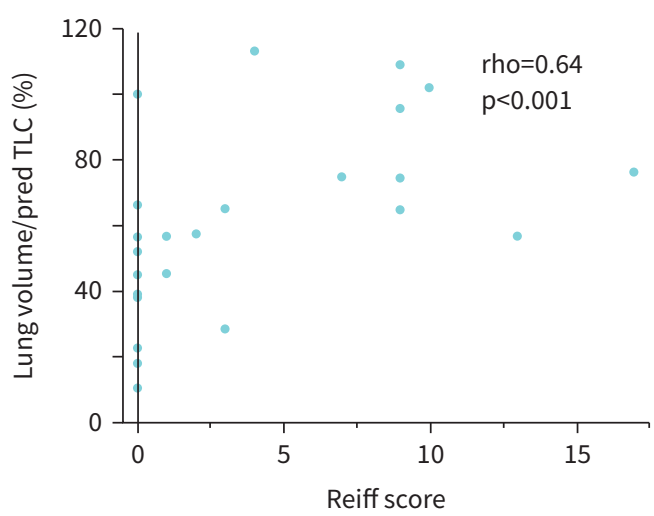

b)

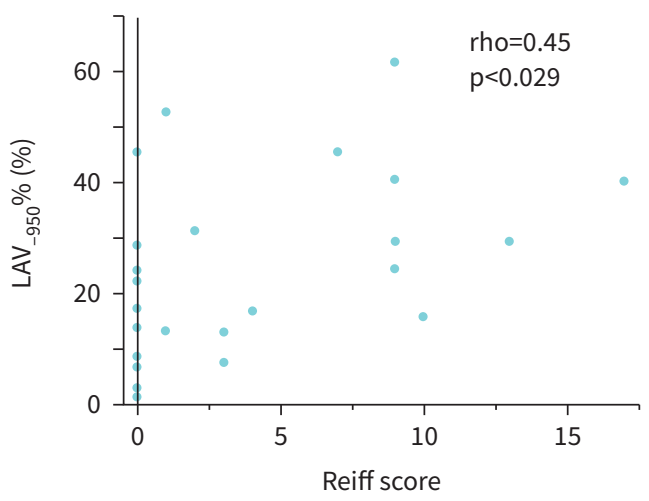

FIGURE 2 Associations between the Reiff score and variables on inspiratory CT. a) CT-TLV/pred TLC and b) $\mathrm{LAV}_{-950} \%$. The extent of airway dilatation was expressed by the Reiff score, which assessed the number of lobes involved (with the lingula considered to be a separate lobe) and the degree of dilatation (tubular=1, varicose $=2$ and cystic $=3$ ). The maximum score is 18 , and the minimum score is 1 for a patient with airway dilatation. Patients without airway dilatation were scored as 0 . CT: computed tomography; pred TLC: predicted total lung capacity; LAV $_{-950} \%$ : percentage of low attenuation volume $<-950$ Hounsfield units.

\section{Discussion}

To the best of our knowledge, this is the first study to demonstrate associations between central airway dilatation on CT and pathological severity of BO as well as air trapping in patients with BO. Furthermore, central airway dilatation, which was observed in $58 \%$ of $\mathrm{BO}$ patients in this study, was associated with isolations of $P$. aeruginosa from the airway.

Airway dilatation on CT in patients with BO has been studied since the 1990s [8, 11-16]. GazouRIAN et al. [15] recently refocused on the importance of central airway dilatation by showing its association with air trapping represented as RV/TLC. With our results of increased \%RV and CT-TLV/pred TLC in patients with airway dilatation, the relationship between the presence of airway dilatation and air trapping in $\mathrm{BO}$ was confirmed. We further demonstrated that airway dilatation was significantly associated with $\mathrm{LAV}_{-950} \%$ and marginally with $\mathrm{fSAD}$, an index of air trapping by CT analysis, using a nonrigid registration technique or parametric response mapping. We also showed that the pathological progression of $\mathrm{BO}$ could be one of the underlying mechanisms of central airway dilatation recognised on CT; the presence of airway dilatation indicated that $62 \%$ of bronchioles were affected with a BO median of 1.6 months later with a sensitivity of $92 \%$ and specificity of $60 \%$. Thus, the present data extended the previous findings by clearly showing an association between the development of airway dilatation and the histopathological extent of BO. Patients with airway dilatation had a higher proportion of emphysema-like lesions detected by CT than those without airway dilatation. Of interest, as shown in Suppl. Figure 2A, in BO patients the emphysema-like lesion was homogeneously distributed, and the secondary pulmonary lobule was not well-defined. This spatial pattern is different from the centrilobular emphysema commonly found in smokers with COPD, which is radiologically characterised by well-defined low attenuation regions surrounded by normal lung regions. (Suppl. Figure 2B). These findings suggest that the reduction in lung tissue density revealed by CT in BO patients is not associated with parenchymal destruction. We speculate that the relatively increased collateral ventilation due to reduced direct ventilation from conducting airway on parenchyma [25] and the regional hypoxic vasoconstriction due to local hypoxia might decrease local tissue density [26], which could be recognised by CT as a low attenuation region. Furthermore, impaired lung development may also induce insufficient alveolisation and reduce lung tissue density and CT values in these regions. WAsнко et al. [27] examined patients from 18 to 30 years old until their middle age, demonstrating that a failure to reach the predicted level of peak $\mathrm{FEV}_{1}$ or accelerated decline in $\mathrm{FEV}_{1}$ after that, was associated with the development of airspace dilatation, which was considered a result of early life inflammatory and immunological events. Although further study is needed to clarify the relationship between emphysema-like lesion and airway dilatation in BO, this study is one of the first to focus on emphysema in association with airway dilatation in $\mathrm{BO}$.

In previous studies of BO, the prevalence of airway dilatation varies widely, between $11 \%$ and $71 \%$ [8, 12, 15], which may be due to differences in timing of its detection from the diagnosis of $\mathrm{BO}$ as well as disease 
a)

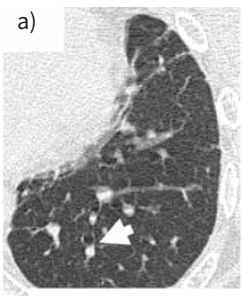

Lumen $4.5 \mathrm{~mm}^{2}$

Before the diagnosis of $\mathrm{BO}$

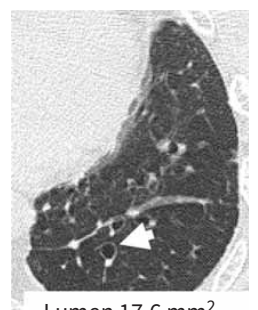

Lumen $17.6 \mathrm{~mm}^{2}$

At the time of diagnosis of $\mathrm{BO}$

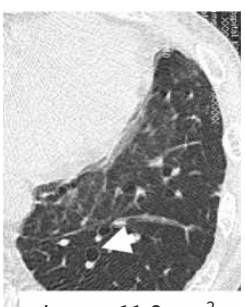

Lumen $11.6 \mathrm{~mm}^{2}$

At the time of registration for lung transplantation
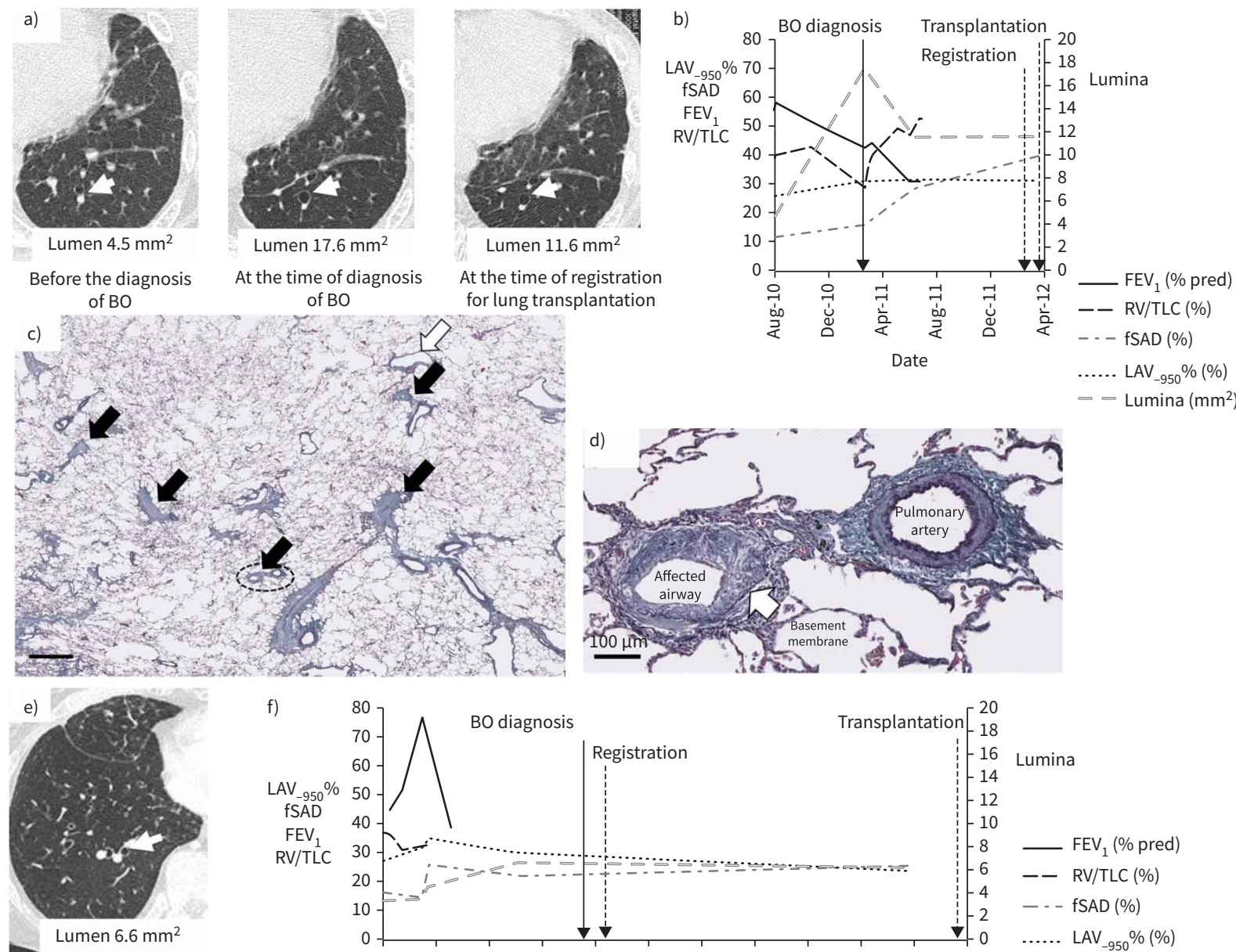

At the time of registration for lung transplantation
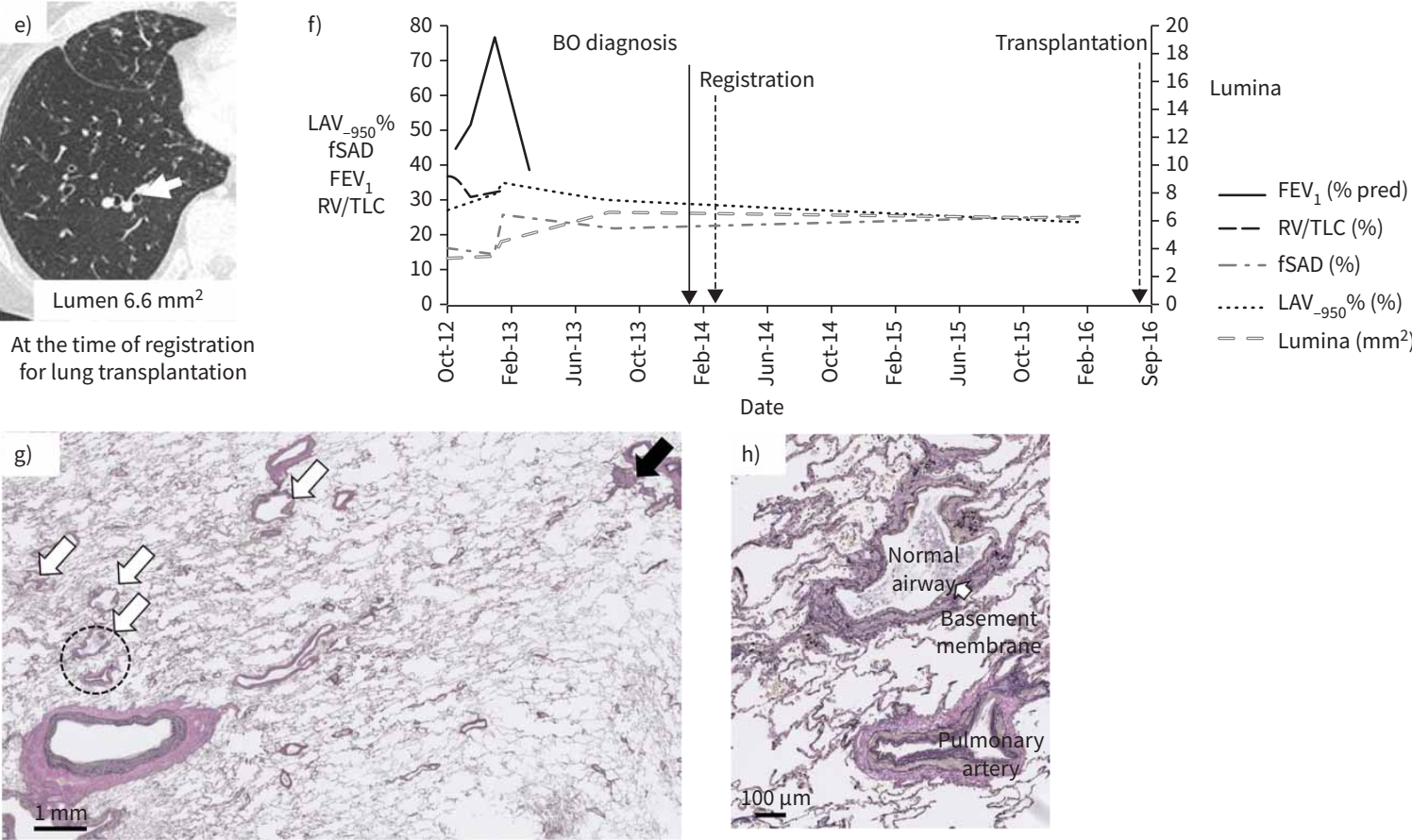

FIGURE 3 Longitudinal data of patients with $(a-d)$ and without $(e-h)$ airway dilatation. a) and e) show HRCT of each patient. The airways with a white arrow were measured for lumen. The longitudinal changes in pulmonary function test (unavailable from the middle due to repeating pneumothorax in both patients) and indices on HRCT are presented in (b) and (f). c) and g) show representative lung histology of the patients. The small airways $<2 \mathrm{~mm}$ in diameter are shown with arrows (white arrow: normal airway, black arrow: narrowing or obstructive airway). [c) elastica Masson stain, g) elastica van Gieson stain]. d) and h) show one of the narrowing and normal airways, respectively, at high magnification of circled airways in (c) and (g) [d) elastica Masson stain, h) elastica van Gieson stain]. BO: bronchiolitis obliterans; FEV ${ }_{1}$ : forced expiratory volume in $1 \mathrm{~s}$; HRCT: high-resolution computed tomography; LAV $_{-950} \%$ : percentage of low attenuation volume <-950 Hounsfield units; fSAD: functional small airway disease; RV: residual volume; TLC: total lung capacity.

severity of BO; one study that reported the lowest prevalence of airway dilatation included patients around only 1 year after the diagnosis of BO, which was about half the time of 2.1 years in our cohort [12]. Indeed, GAzouRian et al. [16] reported deterioration of airway dilatation over time after the diagnosis of 
a)

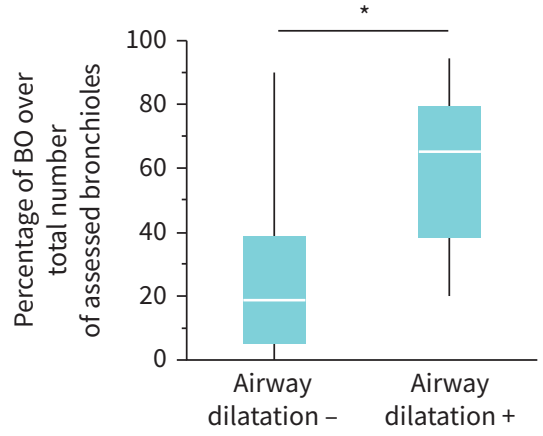

c)

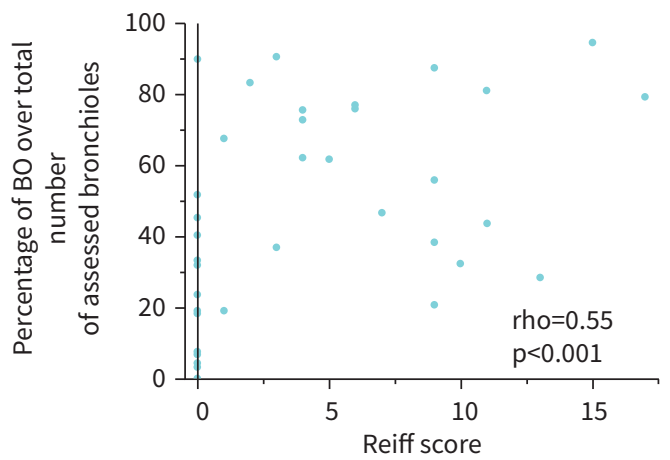

b)
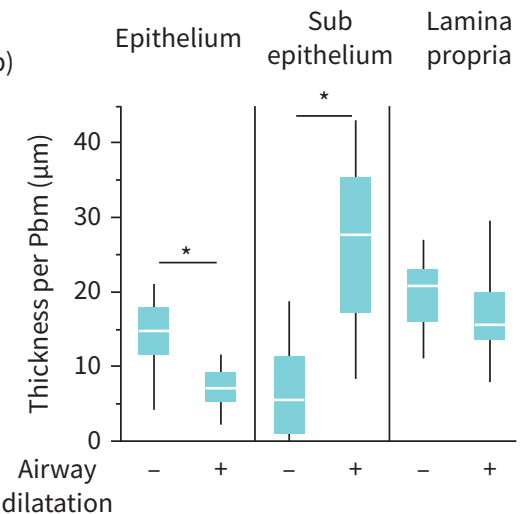

d)

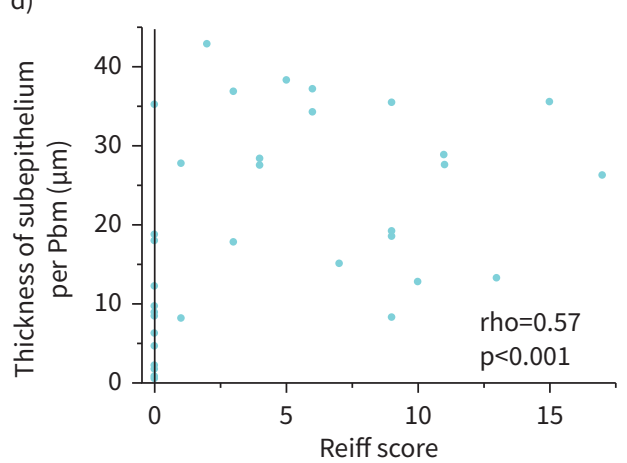

FIGURE 4 Pathological measurements related to BO of relatively circular bronchioles. a) The percentage of narrowing or obstructive bronchioles due to $\mathrm{BO}$ among the total number of assessed bronchioles per patient and b) thickness of airway wall compartments adjusted by Pbm. c) and d) show the associations between Reiff score and the extent of BO and thickness of subepithelium. The Reiff score assesses the number of lobes involved (with the lingula considered to be a separate lobe) and the degree of dilatation (tubular=1, varicose $=2$ and cystic=3). The maximum score is 18 , and the minimum score is 1 for a patient with airway dilatation. Patients without airway dilatation were scored as 0 . The boxes represent the interquartile range with a median (the horizontal line). BO: bronchiolitis obliterans; Pbm: perimeter of the basement membrane. ${ }^{*} p<0.05$.

$\mathrm{BO}$ in patients with a progressive decline in $\mathrm{FEV}_{1}$, although they did not assess pathologies. Additionally, the ROC analysis in this study showed the relationship between airway dilatation on CT and pathologically extensive BO. We concluded that the development of airway dilatation might be a phenomenon occurring in the relatively progressive or late phase of BO. It might be more meaningful during follow-up of $\mathrm{BO}$, such as better allocation for lung (re-)transplantation, than for the early diagnosis.

The mechanisms underlying the associations between central airway dilatation and the progression of BO remain unclear. When considering our result of the decreased epithelial thickness of bronchioles in patients with prominent airway dilatation, one possible explanation is that severe and irreversible injury due to chronic graft-versus-host disease denuded epithelium and caused chronic low-grade inflammation of the airways, which resulted in an architectural weakening of proximal airway walls [28, 29]. The microbiome change during the progression of $\mathrm{BO}$, inducing colonisation of $P$. aeruginosa, could be the second hit for developing airway dilatation $[1,29,30]$.

The assessment of airway dilatation is thus essential. Meanwhile, there is no consensus in the valid scoring system for the extent of airway dilatation in BO patients. Previous studies of BO calculated the mean diameter of the airway lumen using specific software $[15,16]$, while we adopted the modified Reiff score, which is often used in the evaluation of bronchiectasis. A previous report on bronchiectasis showed that the extent of bronchiectasis graded similarly using the Reiff score was significantly associated with airflow limitation, RV and reduction in gas transfer, independently of airway wall thickening and air trapping on CT. At the same time, these associations were not observed for the diameter of the dilated airways [26]. 
Therefore, we consider the Reiff score to be a useful and clinically applicable index for grading the extent of airway dilatation in $\mathrm{BO}$.

Several limitations should be addressed. First, this is a historical cohort study with missing data, in particular, data on HRCT for quantitative analysis and pulmonary function. Indeed, the results of pulmonary function tests at registration were available only for $53 \%$ of our cohort due to their severe respiratory conditions or recurrent pneumothorax. The results of gas transfer, which are important to assess emphysema physiologically, were also lacking in most patients. Second, the small sample size from a single centre made it difficult to evaluate confounding factors that may have affected the results. However, this study is the first to statistically evaluate the pathologies of $\mathrm{BO}$, particularly in association with airway dilatation. Moreover, we could confirm our results in a subgroup of patients who received living-donor lung transplantation, who had only a small time gap between CT evaluation and pathology. Further studies will be required to clarify the mechanisms underlying the association between central airway dilatation and the progression of BO. Although these limitations warrant careful interpretation of our results, the clinical, radiological and pathological findings of this study re-emphasize the importance of airway dilatation recognition in the management of $\mathrm{BO}$. This would be particularly important because many patients with severe BO repeat pneumothorax in their clinical courses, which hamper pulmonary function tests.

\section{Conclusions}

Central airway dilatation on CT may reflect the histopathological extent of BO and isolations of Pseudomonas aeruginosa from the airway, emphasizing the importance of its recognition in the management of BO. The mechanisms underlying the associations between central airway dilatation and the development of BO may need further study.

Acknowledgement: The authors want to thank Maki Isomi for coordinating lung transplants.

Provenance: Submitted article, peer reviewed.

Conflict of interest: M. Kogo has nothing to disclose. H. Matsumoto has nothing to disclose. N. Tanabe reports grants from Fujifilm during the conduct of the study; and personal fees from AstraZeneca, Nippon Boehringer Ingelheim and GlaxoSmithKline outside the submitted work. T.F. Chen-Yoshikawa has nothing to disclose. N. Nakajima has nothing to disclose. A. Yoshizawa has nothing to disclose. T. Oguma reports research grants from Fujifilm Corporation, and lecture fees from AstraZeneca, KYORIN Pharmaceutical, GlaxoSmithKline and Novartis, outside the submitted work. S. Sato reports grants from Nippon Boehringer Ingelheim outside the submitted work. N. Nomura has nothing to disclose. C. Morimoto has nothing to disclose. H. Sunadome has nothing to disclose. S. Gotoh reports grants from Kyorin Pharmaceutical Co. Ltd, Fujifilm Wako Pure Chemical Corp. and the Japan Agency for Medical Research and Development, and is a founder of and shareholder in HiLung. Inc., outside the submitted work. A. Ohsumi has nothing to disclose. H. Date has nothing to disclose. T. Hirai has nothing to disclose.

\section{References}

$1 \quad$ Aguilar PR, Michelson AP, Isakow W. Obliterative bronchiolitis. Transplantation 2016; 100: 272-283.

2 Barker AF, Bergeron A, Rom WN, et al. Obliterative bronchiolitis. N Engl J Med 2014; 370: 1820-1828.

3 Chen-Yoshikawa TF, Sugimoto S, Shiraishi T, et al. Prognostic factors in lung transplantation after hematopoietic stem cell transplantation. Transplantation 2018; 102: 154-161.

4 Verleden SE, Sacreas A, Vos R, et al. Advances in understanding bronchiolitis obliterans after lung transplantation. Chest 2016; 150: 219-225.

5 de Jong PA, Dodd JD, Coxson HO, et al. Bronchiolitis obliterans following lung transplantation: early detection using computed tomographic scanning. Thorax 2006; 61: 799-804.

6 Belloli EA, Degtiar I, Wang X, et al. Parametric response mapping as an imaging biomarker in lung transplant recipients. Am J Respir Crit Care Med 2017; 195: 942-952.

7 Cheng GS, Selwa KE, Hatt C, et al. Multicenter evaluation of parametric response mapping as an indicator of bronchiolitis obliterans syndrome after hematopoietic stem cell transplantation. Am J Transplant 2020; 20: 2198-2205.

8 Gunn ML, Godwin JD, Kanne JP, et al. High-resolution CT findings of bronchiolitis obliterans syndrome after hematopoietic stem cell transplantation. J Thorac Imaging 2008; 23: 244-250.

9 Song I, Yi CA, Han J, et al. CT findings of late-onset noninfectious pulmonary complications in patients with pathologically proven graft-versus-host disease after allogeneic stem cell transplant. AJR Am J Roentgenol 2012; 199: 581-587. 
Galban CJ, Boes JL, Bule M, et al. Parametric response mapping as an indicator of bronchiolitis obliterans syndrome after hematopoietic stem cell transplantation. Biol Blood Marrow Transplant 2014; 20: $1592-1598$.

11 Leung AN, Fisher K, Valentine V, et al. Bronchiolitis obliterans after lung transplantation: detection using expiratory HRCT. Chest 1998; 113: 365-370.

12 Ooi GC, Peh WC, Ip M. High-resolution computed tomography of bronchiolitis obliterans syndrome after bone marrow transplantation. Respiration 1998; 65: 187-191.

13 Lentz D, Bergin CJ, Berry GJ, et al. Diagnosis of bronchiolitis obliterans in heart-lung transplantation patients: importance of bronchial dilatation on CT. AJR Am J Roentgenol 1992; 159: 463-467.

14 Sargent MA, Cairns RA, Murdoch MJ, et al. Obstructive lung disease in children after allogeneic bone marrow transplantation: evaluation with high-resolution CT. AJR Am J Roentgenol 1995; 164: 693-696.

15 Gazourian L, Coronata AM, Rogers AJ, et al. Airway dilation in bronchiolitis obliterans after allogeneic hematopoietic stem cell transplantation. Respir Med 2013; 107: 276-283.

16 Gazourian L, Ash S, Meserve EEK, et al. Quantitative computed tomography assessment of bronchiolitis obliterans syndrome after lung transplantation. Clin Transplant 2017; 31: 12943.

17 Bedi P, Chalmers JD, Goeminne PC, et al. The BRICS (Bronchiectasis Radiologically Indexed CT Score): a multicenter study score for use in idiopathic and postinfective bronchiectasis. Chest 2018; 153: 1177-1186.

18 Chalmers JD, Goeminne P, Aliberti S, et al. The bronchiectasis severity index. An international derivation and validation study. Am J Respir Crit Care Med 2014; 189: 576-585.

19 Quanjer PH, Tammeling GJ, Cotes JE, et al. Lung volumes and forced ventilatory flows. Eur Respir J 1993; 6: Suppl. 16, 5-40.

20 Bhatt SP, Soler X, Wang X, et al. Association between functional small airway disease and FEV 1 decline in chronic obstructive pulmonary disease. Am J Respir Crit Care Med 2016; 194: 178-184.

21 Takeuchi Y, Miyagawa-Hayashino A, Chen F, et al. Pleuroparenchymal fibroelastosis, and non-specific interstitial pneumonia: frequent pulmonary sequelae of haematopoietic stem cell transplantation. Histopathology 2015; 66: 536-544.

22 Hogg JC, Chu F, Utokaparch S, et al. The nature of small-airway obstruction in chronic obstructive pulmonary disease. N Engl J Med 2004; 350: 2645-2653.

23 Zheng PP, van der Weiden M, Kros JM. Fast-tracking of co-localization of multiple markers by using the Nanozoomer slide scanner and NDPViewer. J Cell Physiol 2014; 229: 967-973.

24 Tanabe N, Vasilescu DM, Kirby M, et al. Analysis of airway pathology in COPD using a combination of computed tomography, micro-computed tomography, and histology. Eur Respir J 2018; $51: 1701245$. Roberts HR, Wells AU, Milne DG, et al. Airflow obstruction in bronchiectasis: correlation between computed tomography features and pulmonary function tests. Thorax 2000; 55: 198-204.

27 Washko GR, Colangelo LA, Estepar RSJ, et al. Adult life-course trajectories of lung function and the development of emphysema: The CARDIA lung study. Am J Med 2020; 133: 222-230.e211.

King PT. The pathophysiology of bronchiectasis. Int J Chron Obstruct Pulmon Dis 2009; 4: 411-419.

Whitwell F. A study of the pathology and pathogenesis of bronchiectasis. Thorax 1952; 7: 213-239.

Richardson H, Dicker AJ, Barclay H, et al. The microbiome in bronchiectasis. Eur Respir Rev 2019; 28: 190048. 